\title{
ACTITUDES AMBIENTALES EN LOS ESTUDIANTES DE NIVEL SUPERIOR EN MÉXICO
}

\author{
CATALINA VARGAS RAMOS ${ }^{1}$, \\ JUANA MEDELLÍN MORENO², \\ LAURA VÁZQUEZ GALINDO ${ }^{3}$, \\ GUSTAVO GUTIÉRREZ SÁNCHEZ ${ }^{4}$ \\ cathy_vargas@hotmail.com
}

Manizales, 2011-09-12 (Rev. 2011-10-17)

\section{RESUMEN}

La degradación y el consumo excesivo de los recursos naturales nos han llevado a tener problemas ambientales, siendo principalmente problemas por la falta de actitud o de sensibilización ambiental de la población. La palabra ambiental se empieza a escuchar por primera vez en 1975 en la Conferencia de la Organización de las Naciones Unidas (ONU), celebrada en Estocolmo (Suecia), en donde se reconoció la gravedad de estos problemas ambientales surgiendo un tema importante para poder cambiar estas actitudes o cambio de pensamiento a favor del medio ambiente y la necesidad de tener más Educación Ambiental (EA).

En México la EA tuvo su inicio muchos años después en comparación a otros países, siendo el objetivo principal EA formar valores, actitudes, conductas para llegar a tener conciencia a favor del medio ambiente. Por lo que en el ámbito educativo se ha tenido que empezar a abarcar estos temas, teniendo una forma operativa entre la realidad y el medio ambiente, cosa que antes no formaba parte del currículum, ni transversal ni longitudinalmente.

Se pretende con la EA que el estudiante conozca su entorno, tanto en las acciones humanas como en los fenómenos de la naturaleza y que para que esto suceda se requiere enseñarle en forma práctica y en forma teórica como aspectos fisicoquímicos y factores sociales. $Y$ considerando que la educación de hoy, debe de ser innovadora formando a estudiantes con una visión y un criterio amplio para ofrecer resultados y propuestas para la mejora al cuidado del medio ambiente.

\section{PALABRAS CLAVE:}

Actitudes ambientales, ambiental, Educación Ambiental.

\section{ENVIRONMENTAL ATTITUDES IN HIGHER EDUCATION STUDENTS IN MEXICO}

\section{ABSTRACT}

Degradation and overconsumption of natural resources have led to environmental problems, mainly problems due to the lack of the population's attitude or environmental awareness. The word environment was heard for the first time in 1975 at the United Nations Conference (UN) held in Stockholm, Sweden, where the seriousness of environmental problems was recognized and an important issue in order to change those attitudes or ways of thinking in favor of the environment and the need for more Environmental Education (EE) emerged..

EE in Mexico, had its start many years later compared to other countries being its main purpose to educate in values, attitudes, behaviors in order 
to generate consciousness for the environment. This is why in the educational field these issues have been now taken into consideration using an operational way between reality and environment which did not make a part of the curriculum before neither in a crosscurricular nor in a lengthwise way.

EE is intended to introduce students to their environment, both in human actions as in natural phenomena and, for this to happen, it is required to teach students in a practical and theoretical way, as well as in physicochemical aspects and social factors. Also considering that education today must be innovative, educating students with a broad discernment to offer results and proposals for improving the care of the environment.

\section{KEY WORDS:}

Environmental attitudes, environmental, Environmental Education.

\section{INTRODUCCIÓN}

Las profundas contradicciones socioeconómicas, ecológicas y existenciales que arroja como resultado el modelo de desarrollo basado en el crecimiento desmedido y en el anhelo del progreso a nivel macroscópico, hacen complejo conjeturar que sea probable conseguir para los países como México, los beneficios de este tipo de sociedad dentro del actual paradigma tecnológico, mucho menos de las actuales formas de organización de la sociedad (Villegas \& López, 2006). Nuestro país enfrenta un deterioro ambiental en los recursos naturales debido quizá a la expansión de las actividades agrícolas, ganaderas, forestales y pesqueras, por lo que es necesaria una estrategia de educación que logre un equilibrio entre el hombre y la naturaleza (Sosa, Isaac-Márquez, Eastmond, Ayala \& Arteaga, 2010) fomentando la Conciencia Ambiental (CA) entre las nuevas generaciones (Gomera, 2008). Por tanto para interiorizarla se necesita de una Educación Ambiental (EA) que, de acuerdo con Lapera (s.f), implica un proceso de carácter educativo, que además establezca esquemas cognitivos respecto a valores y actitudes, que se manifiesten en comportamientos en favor del cuidado y protección del medio ambiente.

Actualmente la EA tiene como objetivo fundamental formar ciudadanos comprometidos, que tengan ética ambiental y que puedan comprender la relación que existe entre el ser humano y el medio ambiente. De igual forma el de informar acerca del ambiente y de todos sus problemas para una toma de decisión correcta, buscando un equilibrio entre las necesidades a largo o corto plazo. Pero para tener una EA primeramente tenemos que tener un carácter educativo, que va a ir dirigido a formar valores, actitudes, modos de actuación y conductas todas dirigidas a favor del cuidado del medio ambiente. Por tanto la EA de acuerdo a la percepción de los mismos estudiantes puede ser promovida en los centros educativos, especialmente el nivel superior con el fin de que ellos puedan generar un concepto más amplio de lo que la EA implica, esto en el entendido de que en su formación en niveles educativos previos, la enseñanza hacia la EA es limitada y en el peor de los casos hasta nula (Sáez, 1999; Sosa et al., 2010). Por otro lado Villegas y López (2006) hacen una exhortación a reflexionar acerca de la formación universitaria, lo que implica una responsabilidad social y por tanto un desafío para las instituciones de educación superior, por lo que a esta corresponde un involucramiento más dinámico en la formación de profesionistas que 
posean mayor capacidad de análisis a la hora de emprender acciones que puedan involucrar al medio ambiente (Medina, s.f)

Por lo que ahora en el ámbito educativo se ha tenido que empezar a abordar estos temas, teniendo una forma operativa entre la realidad y el medio ambiente, cosa que antes no formaba parte del currículum ni transversal ni longitudinalmente. Se pretende que con la EA el estudiante conozca su entorno, tanto en las acciones humanas como en los fenómenos de la naturaleza y que para que esto suceda se requiere enseñanza integral oportuna, con base en el aprendizaje mediante conferencias de sensibilización, elaboración de cuentos, formación de comités ecológicos, discusiones en el aula y la confección de bitácoras ambientales, todas teniendo en común que el estudiante comprenda y conozca la problemática ambiental y pueda reflexionar y dar soluciones a estos (Espejel \& Castillo, 2008). Y considerando que la educación de hoy debe de ser innovadora formando a estudiantes con una visión y un criterio amplio para ofrecer resultados y propuestas para la mejora del cuidado del medio ambiente, y siendo la EA vista por los educadores como una cuestión ambiental, y por los ambientalistas como una cuestión de enseñanza.

Por ello las personas que desarrollan una labor profesional asumen una tarea protagónica en el desarrollo humano y ambiental sostenible, pues mediante su conocimiento teórico y práctico están capacitados para proponer y ejecutar acciones responsables sobre la base de la ciencia y la tecnología, lo que hace necesario que estén basadas en la ética ambiental específicamente aquella que vela por la vida y no por la destrucción (Hernández et al., 2006). Por lo anterior, el objetivo de este estudio fue conocer las actitudes ambientales que presentan los estudiantes de las carreras de Enfermería, Psicología, y Seguridad, Salud y Medio Ambiente.

Se hizo una evaluación actitudinal en estudiantes del Nivel Superior orientada a cuatro dimensiones ambientales: a) Consumista derrochador, que implica la acumulación, compra o consumo de bienes y servicios considerados no esenciales. b) Consumista consciente, definida como la acción de compra o consumo de bienes no tan esenciales, que se pueden suprimir sin ninguna consecuencia. c) Ecologista bien encaminado, define a aquella persona que procura el cuidado del medio ambiente, pero no reflexiona ante un impacto ambiental, por lo cual podría decirse que le falta la actitud traducida a la acción. d) Ecologista cuidadoso con la madre Tierra, persona que defiende activamente el medio ambiente y reflexiona ante un impacto ambiental y hace uso de sus valores, capacidades y actitudes ambientales necesarias para que se pueda desarrollar un hábito en el cuidado de los recursos naturales.

\section{MÉTODO}

\section{Muestra}

La muestra estuvo constituida por 377 estudiantes universitarios mexicanos de ambos sexos de un rango de edad de 15 a 47 años de las carreras de Enfermería, Psicología, y Salud, Seguridad y Medio Ambiente (SSMA).

\section{Instrumento}

Se trató de una escala Likert compuesta por 11 ítems, con tres opciones de respuesta que van desde 'Muy de acuerdo' hasta 'En desacuerdo', que 
mide la variable actitud ambiental, la cual contiene cuatro dimensiones: a) Consumista derrochador, b) Consumista consciente, c) Ecologista bien encaminado y d) Ecologista cuidadoso con la madre Tierra. Además se valoraron variables socio-demográficas, las cuales fueron: sexo, edad, turno y semestre.

\section{Procedimiento}

Se aplicó el cuestionario en forma colectiva, para lo cual se acudió a las aulas para la recolección de los datos y se les dio las instrucciones. El tiempo de aplicación fue en rango de tiempo de 15 minutos hasta 25 minutos, la aplicación fue anónima y la participación voluntaria. La tabulación de datos se realizó mediante el paquete estadístico SPSS versión 17.0

\section{Resultados}

Los resultados, correspondientes del total de alumnos por carrera, evidenciaron que el $45,6 \%$ correspondió a la carrera de Psicología, seguido del $28,1 \%$ para los estudiantes de la carrera de Seguridad, Salud y Medio Ambiente, y por último el $26,3 \%$ de la carrera de Enfermería, lo cual se muestra en la Tabla 1. Se observa en la Tabla 2 y Tabla 3 , que la actitud 'Ecologista bien encaminado' estuvo mayormente representada con el $75,3 \%$, seguida del $22,5 \%$ para 'Ecologista cuidadoso de la madre Tierra', y por último con el 2,1\% 'Consumista consciente'. El rango de edades con porcentaje más alto se encontró entre los 15-20 años; teniendo una actitud ambiental muy buena ya que fue 'Ecologista bien encaminado' y casi el 19\% 'Ecologista cuidadoso con la madre Tierra', por lo que se vislumbra que en los alumnos existe una EA sólida, por lo que se podría decir que estos alumnos poseen conocimientos sobre la conservación y el cuidado del medio ambiente. Además se obtuvo un cálculo de Chi-cuadrado de Pearson con un valor de 8,974, p >0,0617, el cual indica que no existen diferencias entre la actitud de los alumnos respecto a su carrera profesional.

Tabla 1.Cantidades y porcentajes correspondientes del total de alumnos por carrera

\begin{tabular}{|l|c|}
\hline Carrera & Porcentaje (\%) \\
\hline Enfermería & 26,3 \\
Psicología & 45,6 \\
Seguridad, Salud y Medio Ambiente & 28,1 \\
\hline Total & 100,0 \\
\hline
\end{tabular}

Tabla 2.Cantidad y porcentaje de los alumnos de acuerdo a las actitudes evaluadas.

\begin{tabular}{|l|c|c|}
\hline Actitud & Cantidad & Porcentaje (\%) \\
\hline Consumista consciente & 8 & 2,1 \\
\hline Ecologista bien encaminado & 284 & 75,3 \\
\hline $\begin{array}{l}\text { Ecologista cuidadoso con la } \\
\text { madre Tierra }\end{array}$ & 85 & 22,6 \\
\hline Total & 377 & 100,0 \\
\hline
\end{tabular}

Tabla 3.Cantidad de alumnos de acuerdo a la actitud y carrera correspondiente.

\begin{tabular}{|l|c|c|c|c|}
\hline Carrera & $\begin{array}{c}\text { Consumista } \\
\text { consciente }\end{array}$ & $\begin{array}{c}\text { Ecologista bien } \\
\text { encaminado }\end{array}$ & $\begin{array}{c}\text { Ecologista } \\
\text { cuidadoso con la } \\
\text { madre Tierra }\end{array}$ & Total \\
\hline Enfermería & 4 & 70 & 25 & 99 \\
\hline Psicología & 3 & 140 & 29 & 172 \\
\hline SSMA & 1 & 74 & 31 & 106 \\
\hline Total & 8 & 284 & 85 & 377 \\
\hline
\end{tabular}




\section{DISCUSIÓN}

De acuerdo con lo obtenido en el presente estudio respecto a la actitud ambiental 'Ecologista bien encaminado' y 'Ecologista cuidadoso con la madre Tierra', se puede vislumbrar que en los alumnos existe una EA, por lo que se podría decir que estos alumnos poseen conocimientos sobre la conservación y el cuidado del medio ambiente y que pueden ser portavoces para nuevas generaciones (Hernández et al., 2006). Es importante que se continúe con la promoción de la EA, sobre todo en los centros educativos de nivel superior (Villegas \& López, 2006) y se haga énfasis acerca de los beneficios que se obtienen al cuidar el medio ambiente y sus recursos a través de programas que hasta ahora han dado buenos resultados, para crear conciencia en las personas acerca de la importancia del medio ambiente para la preservación de la vida en el planeta Tierra. Como Espejel \& Castillo (2008) quienes han afirmado acerca del efecto favorable que promueven las charlas y el desarrollo de temas con los universitarios, donde se remarca su trascendente participación en el sentido de que ellos toman el papel más importante como promotores de la Conciencia Ambiental, permitiéndoles así vislumbrar las positivas consecuencias que surgirán a mediano y largo plazo, luego de realizar actos a favor del cuidado del ambiente.

Los resultados del presente estudio pueden servir para incluir en los planes educativos del nivel superior temas relacionados con la educación ambiental, con el fin de aumentar la presencia del tema en los programas de instituciones públicas (Díaz \& Beerli, 2006), como respuesta a esa responsabilidad que como instituciones educativas tienen en la formación de la EA especialmente del nivel superior en México (Sáez, 1999; Sosa et al., 2010; Vargas, Vázquez, Gutiérrez, Vargas \& Fernández, 2010), y por tanto aumentando el bagaje de conocimiento del medio ambiente. Por lo que la EA debe comenzar introduciendo aspectos ambientales, para que los alumnos obtengan bases científicas mediante las cuales estén fundamentado el conocimiento acerca de la problemática ambiental, y de ahí poder conseguir el desarrollo de la CA y ampliar los marcos de referencia, ir más allá de las bibliografías, y promover entre los estudiantes acciones que lleven a la práctica diaria los valores y actitudes (Espejel \& Castillo, 2008).

\section{BIBLIOGRAFÍA CONSULTADA Y REFERENCIADA}

- Díaz, G., y Beerli, A. (2006). Proceso de adopción de la conducta de reciclado: modelos explicativos y variables moderadoras. Cuadernos de Economía y Dirección de la Empresa, 28, 55-86.

- Espejel, A., y Castillo, M. I. (2008). Educación Ambiental para el nivel medio superior: propuesta y evaluación. Revista Iberoamericana de Educación, 46(2), 1-11. Disponible en: http://www.rieoei.org/expe/2299Espejelv2.pdf

- Gomera, A. (2008). La conciencia ambiental como herramienta para la educación ambiental: conclusiones y reflexiones de un estudio en el ámbito universitario. Disponible en: http://www.marm.es/es/ceneam/articulos-deopinion/2008_11gomera1_tcm7-141797.pdf

- González G., E. (2007). La Educación Ambiental de cara a la problemática ambiental Global. Ciencia UANL, 10(004), 425432.

- Hernández, L. M., Carrillo, M. A, Charpentier, C., Brenes, O. E., García, J., Mata, C., Zúñiga, E., y Arnáez, E. (2006). La dimensión ambiental en el currículo universitario: un proceso de 
cambio en la formación profesional. Revista Electrónica Actualidades Investigativas en Educación, 6(001), 1-23.

- Lapera, B. (s.f.). Estrategia metodológica para la educación en valores ambientales de los estudiantes de Ingeniería Química mediante las clases de inglés. Disponible en: http://www.bibliomaster.com/pdf/5328.pdf

- Medina, L. (s.f.). Ensayo: La Educación Ambiental en el Nivel Superior. Disponible en: http://www.uaemex.mx/plin/psus/rev3/medina.pdf

- Meléndez Dobles, S. (2002). La historia ambiental: aportes interdisciplinarios y balance crítico desde América Latina. Cuadernos digitales: Publicación electrónica en historia, archivística y estudios sociales, 7(19), 1-48.

- Moreno, M., Corraliza, J. A., y Ruiz, J. P. (2005). Escala de actitudes ambientales hacia problemas específicos. Psicothema, 17(3), 502-508.

- Pasek, E. (2004). Hacia una conciencia ambiental. Educere, 8(024), 34-40.

- Rojas, E. (2006). Educación Ambiental en el Siglo XXI. Luna Azul (On Line). Disponible en: http://lunazul.ucaldas.edu.co/index.php?option=com_content\&ta sk=view\&id=178\&ltemid=178

- Sáez, V. (1999). Consideraciones sobre la preparación, valores y creencias de los estudiantes de Geografía de la Universidad Central de Venezuela con respecto a la Educación Ambiental. Terra, 15(024), 117-131.

- Salavarría, O. O., y Márquez, R. I. (s.f.). La formación Ambiental en el nivel medio superior del municipio de Campeche. Disponible en:

http://www.comie.org.mx/congreso/memoria/v9/ponencias/at03/ PRE1178946480.pdf

- Sepúlveda, L. E. (2009). Una evaluación de los procesos educativo-ambientales de Manizales. Luna Azul, 28, 46-56.

- Sosa, S. B., Isaac-Márquez, R., Eastmond, A., Ayala, M. E., y Arteaga, M. A. (2010). Educación superior y cultura ambiental en el sureste de México. Universidad y Ciencia, 26(1), 33-49.

- Terrón, E. (2004). La Educación Ambiental en la educación básica, un proyecto inconcluso. Revista Latinoamericana de Estudios Educativos, 34(004), 107-164.

- Vargas, C., Vázquez, L., Gutiérrez, G., Vargas, A., y Fernández, C. (2010). Conciencia ambiental en estudiantes de Enfermería Básica. Revista Universitaria de Investigación y Diálogos Académicos, 6(3), 18-28.

- Villegas, G., y López, M. A. (2006). ¿Es posible el desarrollo sostenible? Acercamientos Conceptuales a la relación ambiente desarrollo y Economía. Luna Azul, 1-9. Disponible en: http://lunazul.ucaldas.edu.co/downloads/0e6423a6Revista11_12 6.pdf

1. M.C.I.A. Universidad Autónoma de Tamaulipas. E-mail: cathy_vargas@hotmail.com

2. M.A.C. Universidad Autónoma de Tamaulipas. E-mail: jumedellin@uat,edu.mx

3. Dra. Universidad Autónoma de Tamaulipas. E-mail: Ivazquez@uat.edu.mx

4. M.C.E. Universidad Autónoma de Tamaulipas. E-mail: tavo_glz@yahoo.com 